Meta

Journal des traducteurs

Translators' Journal

\title{
Vers un apprentissage semi-autonome du processus de la traduction
}

\section{Danny Masschelein et Walter Verschueren}

Volume 50, numéro 2, avril 2005

Processus et cheminements en traduction et interprétation Processes and Pathways in Translation and Interpretation

URI : https://id.erudit.org/iderudit/011000ar

DOI : https://doi.org/10.7202/011000ar

Aller au sommaire du numéro

Éditeur(s)

Les Presses de l'Université de Montréal

ISSN

0026-0452 (imprimé)

1492-1421 (numérique)

Découvrir la revue

Citer cet article

Masschelein, D. \& Verschueren, W. (2005). Vers un apprentissage semi-autonome du processus de la traduction. Meta, 50(2), 560-572. https://doi.org/10.7202/011000ar
Résumé de l'article

Il est difficile d'entamer le débat sur l'évaluation de la traduction si on ne précise pas dans quel contexte (professionnel ou didactique) celle-ci va être pratiquée ou quelle est sa fonction exacte (stimuler les apprenants à perfectionner leurs compétences et performances ou sanctionner un produit final). Cet article contient, donc, une description du cadre local dans lequel nous avons développé une méthode d'évaluation qui met à profit les avantages de tout un éventail d'outils électroniques (en premier lieu, le logiciel d'annotation Markin) et qui tente de concilier les exigences de l'évaluation formative et de l'évaluation sommative, tout en privilégiant la première. Le contexte didactique conditionne les réponses qu'on donne à des questions classiques, comme celles qui ont trait au statut de la faute de langue dans les cours de traduction, ou à l'importance relative de la cause et de l'effet des erreurs et des fautes.
Ce document est protégé par la loi sur le droit d'auteur. L'utilisation des services d'Érudit (y compris la reproduction) est assujettie à sa politique d'utilisation que vous pouvez consulter en ligne.

https://apropos.erudit.org/fr/usagers/politique-dutilisation/ 


\title{
Vers un apprentissage semi-autonome du processus de la traduction
}

\author{
DANNY MASSCHELEIN \\ Hogeschool voor Wetenschap en Kunst, Bruxelles, Belgique \\ dmassche@vlekho.wenk.be \\ WALTER VERSCHUEREN \\ Hogeschool voor Wetenschap en Kunst, Bruxelles, Belgique \\ wverschu@vlekho.wenk.be
}

\begin{abstract}
RÉSUMÉ
Il est difficile d'entamer le débat sur l'évaluation de la traduction si on ne précise pas dans quel contexte (professionnel ou didactique) celle-ci va être pratiquée ou quelle est sa fonction exacte (stimuler les apprenants à perfectionner leurs compétences et performances ou sanctionner un produit final). Cet article contient, donc, une description du cadre local dans lequel nous avons développé une méthode d'évaluation qui met à profit les avantages de tout un éventail d'outils électroniques (en premier lieu, le logiciel d'annotation Markin) et qui tente de concilier les exigences de l'évaluation formative et de l'évaluation sommative, tout en privilégiant la première. Le contexte didactique conditionne les réponses qu'on donne à des questions classiques, comme celles qui ont trait au statut de la faute de langue dans les cours de traduction, ou à l'importance relative de la cause et de l'effet des erreurs et des fautes.
\end{abstract}

\begin{abstract}
Critical discussion on translation quality assessment only makes sense if one takes into full account the professional and didactic contexts in which the assessment takes place and if its precise purpose is specified (is the purpose to stimulate students to perfect their translation competence and performance or to sanction a final product?). This article describes the didactic context in which we have developed a method of translation quality assessment that makes use of an entire range of electronic tools (in the first place the Markin software programme) and that tries to reconcile the need for formative evaluation with the need for summative evaluation - with special emphasis on the first. The didactic context has a significant impact on the responses to a number of classic questions, like those that relate to the status of language mistakes in translation classes or the relative importance of the cause and effect of errors and mistakes.
\end{abstract}

\section{MOTS-CLÉS/KEYWORDS}

évaluation en traduction, évaluation formative, processus de la traduction, apprentissage électronique

\section{Introduction}

La dernière décennie se caractérise par une certaine prolifération de publications consacrées à la problématique de l'évaluation de la traduction. Cependant, même après avoir exploré un échantillon représentatif des livres et articles qui ont vu le jour, le professeur de traduction qui est à la recherche d'une méthode de correction adéquate peut avoir l'impression de rester quelque peu sur sa faim. En effet, très souvent les auteurs présentent des considérations abstraites sur le sujet, sans spécifier 
si la méthode préconisée doit être appliquée dans un contexte professionnel ou didactique, s'il est question d'une évaluation sommative ou d'une évaluation formative, quelles sont les circonstances didactiques dans lesquelles se déroulent les processus de la traduction et de l'évaluation, etc.

Dans cet article, nous analysons d'abord quelques approches plus ou moins récentes, et nous essayons d'expliciter, si besoin en est, le contexte dans lequel doit être située l'évaluation décrite dans chaque publication. En même temps, nous nous demandons quelle est la place que les auteurs ont réservée respectivement aux fautes de langue et aux fautes de traduction. Dans tous les cas, nous essayons de distiller de la lecture de ces publications plus ou moins théoriques des leçons pour notre pratique didactique. Dans une deuxième étape, nous décrivons les variables concrètes qui déterminent le contexte de travail dans notre département. Ensuite, nous présentons quelques aspects de notre projet didactique; nous mettrons l'accent sur le rôle dévolu au logiciel Markin, que nous utilisons comme outil dans une évaluation fondamentalement formative qui se veut orientée vers un apprentissage semi-autonome, dans un environnement électronique, du processus de la traduction. Pour terminer, nous dédions quelques paragraphes à une réflexion sur le statut scientifique de l'évaluation de la traduction, et nous esquissons quelques projets de recherche empirique.

\section{Les multiples visages de l'évaluation}

Nous n'avons pas l'intention de dresser, dans cet article, un bilan complet de la recherche dans le domaine de l'évaluation de la traduction. Nous renvoyons, pour une description relativement récente de l'état de la question, aux numéros spéciaux des revues The Translator et de META, publiés en 2000 et en 2001 respectivement. Nous nous limitons à examiner brièvement trois publications représentatives de trois courants différents (un modèle axé sur la description linguistique, un plaidoyer masqué pour l'approche fonctionnaliste et une approche proprement pédagogique) afin d'en dégager des conclusions sur la fonction que les auteurs accordent à l'évaluation et sur la typologie d'erreurs qu'ils manient.

\subsection{Le modèle pragmatico-fonctionnel de Juliane House (2001)}

Il est peut-être symptomatique que ni le résumé ni l'introduction de l'article publié par Juliane House dans le numéro spécial de META (2001) ne contiennent une référence aux applications concrètes que l'auteur conçoit pour son modèle d'évaluation. Elle parle de l'évaluation et de la traduction en général; il n'est pas clair si cela implique que son modèle vise à une applicabilité universelle. Le lecteur a de la peine à découvrir si la méthode doit être utilisée dans la critique de traductions et versions générées dans un contexte professionnel, ou plutôt dans un contexte scolaire. Ce n'est qu'après avoir consulté les livres publiés par House $(1977,1997)$ qu'on se rend compte que, au fait, l'auteur n'évalue que des textes délivrés par des traducteurs professionnels, et qu'elle se prononce uniquement sur le texte comme produit, et jamais sur le processus de la traduction.

Ces limitations expliquent, peut-être, pourquoi l'auteur $(1977,1997)$ montre un certain mépris pour les fautes trop apparentes (overtly erroneous errors), comme les fautes de langue et d'interprétation, heureusement peu fréquentes dans les textes 
analysés. Elle a le privilège de pouvoir se concentrer sur des fautes d'un type plus intéressant, moins visibles pour un public non averti (covertly erroneous errors), à savoir, des fautes dites fonctionnelles.

Selon l'auteur, on ne peut parler d'une traduction (un texte qui montre une équivalence sémantique et pragmatique avec le texte original) que si le texte de la langue cible remplit la même fonction que le texte de la langue source (si la fonction est différente, il faut plutôt parler d'une version). House (2001 : 245) critique le concept de «fonction» tel qu'il est employé par les adeptes de l'école du «Skopos»: il faut absolument qu'on propose une définition plus opérationnelle de la notion. Pour House, la fonction d'un texte peut être conçue comme la manière dont celui-ci est employé dans un contexte particulier. D'autre part, elle renonce à étudier le contexte en soi; elle préfère s'en tenir à une analyse purement textuelle, ce qu'elle considère possible, puisque le contexte est encapsulé d'une certaine façon dans le texte, et il suffit de repérer les marques situationnelles visibles dans celui-ci. Or, le traducteur commet une faute fonctionnelle s'il applique mal les soi-disant «filtres culturels». La manière dont une certaine fonction textuelle est réalisée peut être différente d'une culture à l'autre; d'ailleurs, l'auteur a mené des recherches empiriques dans le but de déceler de tels écarts entre les pays germanophones et anglophones. Les traducteurs qui ne respectent pas les conventions textuelles régnant dans la culture du texte cible commettent une faute fonctionnelle, parce qu'ils ont manqué à appliquer un filtre culturel. Cependant, parfois les traducteurs font des péchés par excès, quand ils utilisent un filtre culturel à tort et à travers.

Le titre des monographies $(1977,1997)$ de l'auteur nous semble quelque peu trompeur: en effet, le lecteur est amené à penser que l'essentiel du discours résidera en une réflexion sur un modèle d'évaluation, mais le texte fournit avant tout un arsenal conceptuel intéressant pour la description de textes écrits dans une langue cible déterminée et dérivés d'un texte publié dans une langue différente. La dichotomie «overt translation» (une traduction qui est présentée ouvertement comme telle) versus «covert translation» (un texte qui ne thématise pas son statut de texte dérivé) s'est avérée très intéressante, tout comme la distinction explicite entre traductions et versions. Cependant, dans les textes de House la description linguistique occupe une place bien plus importante que l'évaluation proprement dite, ce que l'auteur semble reconnaître en introduisant, enfin, les mots «linguistic description» dans le titre de son article de META (House 2001). Son mérite consiste à tracer une ligne de démarcation très nette entre l'étape purement descriptive, qui peut aspirer à un certain statut scientifique (car il s'agit d'une démarche intersubjective, contrôlable et reproductible), et, d'autre part, la pratique sociale (et nécessairement subjective) de l'évaluation. En plus, dans un contexte didactique on pourrait essayer de mettre à profit le concept de «filtre culturel» et de demander aux élèves de dupliquer, à une échelle plus modeste, les recherches contrastives de type empirique.

\subsection{Le modèle fonctionnaliste de Jacqueline Hulst}

Le titre de l'article de Jacqueline Hulst (1998) ne peut être plus éloquent: l'auteur n'a d'yeux que pour le «produit» (qui, pour elle, occupe une position «centrale») et elle ne prête pas d'attention à la relevance que l'évaluation pourrait avoir pour le reste du processus de la traduction. D'autre part, dès la première phrase de son texte, elle 
annonce qu'elle va adopter un point de vue didactique dans son commentaire des critères et des méthodes d'évaluation.

L'auteur commence par fustiger (sans qu'elle ose l'avouer, parce qu'elle s'était promis d'adopter une position non normative) les méthodes d'évaluation dites «traditionnelles ", contrastives, d'inspiration philologique, axées sur le texte source. Dans beaucoup de cas, les enseignants qui ont recours à ces procédures antédiluviennes se posent la question, inutile aux yeux de l'auteur, de savoir quel élément est à l'origine de la faute, au lieu de se pencher sur l'effet que le faux pas du traducteur peut avoir sur le fonctionnement du texte cible. Ils ont la mauvaise habitude de tenir compte des connaissances que les élèves sont censés posséder. Aussi, ils attachent une importance majeure à la correction linguistique des textes produits par leurs élèves; souvent, la catégorie linguistique à laquelle appartient l'élément erroné détermine la gravité de la pénalisation (il n'est pas rare que les fautes grammaticales soient considérées plus impardonnables que les fautes lexicales, en vue du prestige de la grammaire dans l'enseignement traditionnel).

Il est évident que Hulst a plus de sympathie pour un deuxième type d'évaluation, de souche fonctionnaliste, centré sur le texte cible, dans lequel les fautes de traduction pèsent bien plus lourd dans la balance que les fautes de langue. L'enseignant se demande tout d'abord si la traduction de ses élèves «fonctionne» comme texte indépendant, c'est-à-dire, si les étudiants sont arrivés à atteindre l'objectif fixé («Skopos»), si le texte est tout à fait acceptable pour le public cible, si on a tenu compte des conventions (textuelles ou autres) de la culture du texte cible, etc. Il ne se pose pas de questions sur les facteurs qui pourraient avoir causé les erreurs, mais sur la mesure dans laquelle ces fautes perturbent le fonctionnement comme texte autonome de la traduction. Une faute fonctionnelle peut être définie comme toute faute qui met en péril la réalisation adéquate des fonctions textuelles, c'est-à-dire, des relations de cohérence et de cohésion. Ce n'est qu'après avoir jugé le texte cible sur ses propres mérites que l'enseignant doit procéder à une confrontation de la traduction avec le texte source. Les déviations sémantiques et structurelles relevées dans cette étape peuvent avoir un effet positif, neutre ou négatif sur le fonctionnement du texte cible.

Hulst (1998: 134) ne commet pas le péché de la naïveté: elle comprend que dans l'enseignement des départements de traduction on ne pourra pas s'abstenir d'aborder aussi des aspects qui ne relèvent pas de la traduction en tant que telle (des fautes dites «stupides», des fautes de langue, enfin, les «overtly erroneous errors» de House). On a beau dire que ce sont des éléments que les élèves devraient avoir appris dans un autre stade de leur formation, ou dans d'autres cours, la réalité nous démontre qu'il est rare que la majorité des élèves d'un centre aient une maîtrise parfaite de leurs langues de travail avant d'entamer des études de traduction.

De prime abord, les instruments d'analyse de Hulst ne semblent pas faciles à appliquer, même dans le cadre de l'évaluation sommative. Un autre aspect discutable, du point de vue didactique, c'est la primauté attribuée au mesurage de l'effet des fautes commises. On s'explique mal comment, en classe, à l'heure de pénaliser certaines erreurs, le professeur ne pourrait pas faire entrer en ligne de compte les connaissances dont l'étudiant dispose à un moment donné de sa formation. À ce propos, il est utile de rappeler la distinction entre les concepts d' «erreur» et de «faute» que l'on fait dans le Cadre européen commun de référence pour les langues: apprendre, enseigner, évaluer (2000: 118) : les erreurs reflètent les compétences qu'un apprenant a acquises 
à un moment donné de son processus d'apprentissage (il existe donc une adéquation entre la compétence et la performance de l'apprenant), pendant qu'une faute peut être attribuée à une mobilisation insuffisante par l'apprenant de ses compétences. En plus, si on conçoit l'évaluation comme une activité qui devrait permettre à l'élève d'améliorer sa performance, il est évident que celui-ci a tout intérêt à comprendre pourquoi il est tombé dans une erreur, et quelles sont les actions qu'il peut entreprendre pour remédier aux carences qui expliquent l'insuffisance provisoire des produits de son travail.

\subsection{Le modèle pédagogique de Hannelore Lee-Jahnke}

L'article de Lee-Jahnke (2001) est une des rares publications à annoncer clairement quel est le cadre dans lequel on doit situer la méthode d'évaluation proposée (un contexte didactique) et quels sont les buts envisagés (à la fois aider les étudiants à s'améliorer dans leurs performances et sanctionner un produit final, en attribuant une note).

L'auteur s'intéresse en priorité à l'évaluation formative, qu'elle présente comme «l'outil par excellence de tout processus d'apprentissage» (Lee-Jahnke 2001: 260). À la différence de Hulst, elle trouve qu'il est important d'identifier les causes des faux pas de ses étudiants. À plusieurs reprises, elle va à la recherche de la «source de leur difficulté» (260), des facteurs qui sont «à l'origine d'erreurs» (263); dans le deuxième volet de son questionnaire d'autoévaluation, les étudiants sont invités à identifier la «source des erreurs» (270). Elle vante les possibilités de l'autoévaluation, qui présuppose la mise en œuvre de stratégies métacognitives, ainsi que de l'évaluation écrite entre pairs. Les mots-clés de son approche semblent être "créativité», «motivation», «sensibilisation», «mise en confiance». Elle signale, en plus, qu'il est primordial que les étudiantes apprennent à manier le métalangage de la discipline.

Lors de l'évaluation sommative, l'auteur distingue trois grandes catégories: l'exactitude (contenu, sens), l'aspect marketing (le «Skopos») et la créativité (la traduction, est-elle idiomatique, ou même artistique?). Chaque catégorie est subdivisée en plusieurs points plus spécifiques, ce qui doit permettre à l'enseignant d'établir une note aussi objective que possible (il peut paraître un peu surprenant que les fautes de langue - orthographe, grammaire, syntaxe - appartiennent à la catégorie de la créativité). En ce qui concerne les deux premières catégories, la traduction est jugée acceptable (et alors l'étudiant obtient le maximum des points, six dans le système suisse) ou pas (dans ce cas, l'enseignant attribue automatiquement la note la moins bonne, c'est-à-dire zéro); l'évaluation de la créativité peut donner lieu à des notes plus nuancées.

\section{Le contexte pédagogique des études de traduction en Belgique}

En Belgique, les étudiants de traduction et d'interprétation (dans le reste de cet article, nous ne parlerons que de la traduction de textes écrits) apprennent leur métier dans une des hautes écoles, associées depuis quelque temps à une des universités du pays. Jusqu'ici, il s'agissait d'une formation de quatre années, qui donnait droit au titre de licencié en traduction. Depuis septembre 2004, un nouveau système remplace ce modèle d'éducation, dans le cadre de l'introduction du système Bachelor-Master (un 
cycle de trois ans menant au grade de bachelier, après quoi les étudiants peuvent parfaire leur formation et obtenir le grade de maître, probablement après un deuxième cycle d'une seule année).

Même dans le passé, les études de traduction réalisées en Belgique, si on les comparait avec des formations du même type fournies dans d'autres pays, se caractérisaient par leur caractère «impur»: en plus de préparer les élèves à une carrière comme traducteur, les départements de traduction leur offraient une solide formation linguistique (en trois langues: la langue maternelle et deux langues étrangères traitées à égalité) et générale. Ils mettaient l'accent, pendant les premières années du programme, sur les cours de langues, au lieu de se concentrer sur les matières liées plus spécifiquement à la traduction.

La réforme du panorama des hautes études ne fera que renforcer la prépondérance des matières de type linguistique, puisque l'organisation modulaire des plans d'études devra faciliter la réorientation des élèves au cours de leurs études (ainsi, il devrait devenir plus aisé pour un élève de traduction de migrer vers une formation d'orientation philologique ou vice versa). Par conséquent, le nombre d'heures de contact consacrées à la traduction dans la phase initiale de l'enseignement diminuera considérablement, et la vraie spécialisation en matière de traduction sera remise jusqu'à la maîtrise. L'évolution décrite ira de pair avec d'autres transformations: le renforcement de la composante académique de la formation des traducteurs (notamment en ce qui concerne l'attention prêtée aux apports de la traductologie) et un glissement progressif d'un modèle d'éducation hiérarchique à prédominance de leçons magistrales vers un nouveau modèle dans lequel l'autonomie de l'apprenant est stimulée et le rôle du professeur devient de plus en plus celui d'un initiateur, médiateur et coach.

\section{L'apprentissage de la traduction dans un environnement électronique}

Les variables pédagogiques mentionnées dans la section précédente nous ont amenés à remodeler foncièrement l'organisation de nos cours de traduction. Étant donné le nombre très restreint d'heures de contact (quinze au total pour la traduction de l'espagnol vers le néerlandais en première année, par exemple), il est évident que les cours présentiels doivent être conçus comme le cercle central autour duquel il faut prévoir toute une série d'activités complémentaires, qui peuvent se dérouler dans un environnement électronique. Pendant les heures qu'ils passent ensemble en classe, les étudiants ont relativement peu d'occasions de mettre en pratique les enseignements du professeur (pourtant, nous prévoyons toujours quelques sessions dans un laboratoire multimédia où nous pouvons observer, à partir de la console centrale du professeur, les tâtonnements des étudiants qui sont en train d'introduire leur traduction, chacun à son propre ordinateur).

Pour pallier cette lacune, les apprenants sont invités à traduire une série de textes exemplaires en dehors des horaires du cours. Les textes à traduire, les consignes et, parfois, un dossier contenant quelques documents parallèles peuvent être téléchargés dans un environnement d'apprentissage électronique (créé, dans notre cas, à l'aide du logiciel Blackboard). Les étudiants remettent leur traduction à travers le même environnement, en format de texte riche (RTF). Dans l'étape suivante, nous introduisons leurs traductions dans le logiciel Markin, que nous présentons brièvement dans la section 3.1, et nous les annotons en nous servant de la barre de boutons 
décrite dans les sections 3.2-3.4. Notre correction écrite des traductions ne doit pas être considérée comme le point final du processus, mais comme un nouveau point de départ, puisque les commentaires insérés à l'aide du logiciel doivent inciter les apprenants à réfléchir sur leur processus de traduction, à comparer les problèmes suscités par le texte traduit avec des difficultés similaires commentées sur une page Web, à recourir à toute une série d'outils, à faire de nouveaux exercices, etc. (voir 3.5).

\subsection{Caractéristiques générales du logiciel Markin}

Le logiciel Markin, qui en est déjà à sa troisième version, a été élaboré par Martin Holmes et peut être téléchargé à la page $<\underline{\text { http://www.cict.co.uk/software/markin/ }}$ index.htm $>$ (il s'agit d'une version démo qui dispose de toutes les fonctionnalités du programme, mais qui permet seulement d'importer des morceaux de texte très courts; pour que le logiciel devienne vraiment rentable, il faut disposer d'un code d'enregistrement que l'on peut acquérir à un prix relativement bas).

À l'aide de ce logiciel, le professeur peut annoter des textes (des compositions ou des traductions, par exemple) d'une façon très efficace. En effet, le programme permet de créer une barre de boutons où chaque bouton représente un type de commentaire (positif ou négatif) dont l'enseignant estime qu'il va être obligé d'introduire à plusieurs reprises. Le professeur assigne à chaque bouton un code, une annotation courte (le texte qui va apparaître dans la correction exportée) et une annotation plus longue (à laquelle l'étudiant pourra accéder en cliquant sur les codes figurant dans la correction exportée, et qui renvoie à une explication plus détaillée au moyen de liens hypertexte) (voir annexe 3); ces commentaires prédéfinis peuvent être classés en groupes (par exemple, des commentaires qui ont trait à l'orthographe, ou au respect de la consigne de traduction, etc.). Une fois que la barre a été configurée, quand le professeur veut identifier une erreur ou louanger une trouvaille extraordinaire, il suffit de cliquer sur un bouton pour ajouter un des commentaires prédéfinis. Lorsque le correcteur se heurte à un phénomène qu'il n'avait pas prévu dans la barre de boutons, il peut toujours ajouter un post-scriptum spécifique (mais même dans ce cas il peut puiser, le cas échéant, dans un inventaire de commentaires réutilisables enregistrés dans une base de données qui est très facile à manipuler); il est également possible d'introduire des commentaires généraux sur la qualité d'ensemble de la production (voir une prise d'écran du menu du logiciel, dans l'annexe 1).

À l'heure de programmer la barre de boutons, l'enseignant a la possibilité d'accorder à chaque bouton un score positif ou négatif. Ensuite, pendant la phase de la correction ou après, il pourra visualiser à chaque instant un résumé des commentaires introduits, du nombre d'erreurs par catégorie, du score total, etc. En plus, il peut générer des statistiques du même type pour un groupe de textes (toutes les traductions qu'un étudiant a remises au cours de l'année, ou toutes les versions d'un même texte, etc.).

Le texte annoté peut être exporté dans plusieurs formats, dont le format HTML nous semble le plus intéressant pour l'apprenant. En effet, cette présentation est très flexible, car elle permet de visualiser l'information disponible avec plus ou moins de détails: l'étudiant peut examiner le texte sur l'écran et suivre les hyperliens pour accéder aux annotations courtes ou longues, mais il peut aussi afficher les codes de correction et imprimer le texte corrigé, ou consulter la liste des codes dans laquelle il 
verra quelle est la pénalisation associée à un certain type de faute, etc. C'est l'enseignant qui décide s'il exporte ou pas les statistiques correspondant aux textes corrigés.

\subsection{Une barre de boutons conçue pour la correction de traductions de l'espagnol vers le néerlandais}

Depuis trois ans, nous utilisons le logiciel Markin dans nos cours de traduction de textes généraux ou spécialisés (textes économiques et financiers) de l'espagnol vers le néerlandais (la langue maternelle des étudiants), et au cours de cette période nous avons adapté et affiné notre barre de boutons à plusieurs reprises.

Dans sa forme actuelle (voir la prise d'écran de l'annexe 2), la barre contient 7 boutons «positifs» et quelque 90 boutons «négatifs». Nous appuyons sur les boutons positifs quand le texte remis par l'étudiant est impeccable du point de vue linguistique, quand le style est particulièrement brillant dans un ou plusieurs passages, quand le texte cible est ajusté d'une façon parfaite à l'objectif de la traduction, quand l'étudiant a su compenser intelligemment la perte de certains éléments esthétiques présents dans le texte source, quand la documentation qu'il a réunie est très étoffée, quand le cahier des charges qu'il a ajouté à sa traduction est bien fait, etc. Les boutons négatifs, quant à eux, ont été subdivisés en sept catégories, desquelles les trois premières se rapportent à des fautes de langue (des fautes d'orthographe et de ponctuation, des fautes lexicales et des fautes morphologiques et syntaxiques), la quatrième à des questions discursives et stylistiques, la cinquième à la présentation du texte et à toute une série de variables contextuelles (ponctualité, format et titre du fichier, etc.), la sixième à l'exactitude et au "Skopos», la septième à l'emploi judicieux des outils disponibles (le contrôle orthographique et grammatical du traitement de texte, la documentation mise à la disposition de l'étudiant dans l'environnement électronique, une base de données de termes économiques et financiers), et la dernière à toute une série de problèmes contrastifs typiques de la combinaison linguistique espagnol/ néerlandais.

Pour rédiger les annotations, nous nous sommes inspirés de la terminologie présentée dans la version néerlandaise de la Terminologie de la traduction (Delisle et. al. 2003).

\subsection{L'importance relative des fautes de langue}

Nous souscrivons à la thèse de ceux qui pensent que dans les cours de traduction il faudrait parler avant tout des problèmes proprement traductologiques. Cela ne veut pas dire, pour autant, que l'enseignant devrait «laisser passer» les fautes d'orthographe et de syntaxe (des imperfections dont on dit parfois qu'elles n'ont pas de répercussions graves sur le fonctionnement du texte), sous le prétexte qu'il s'agit de fautes «stupides» que les élèves ont appris à éviter dans d'autres cours. Après tout, une convention non écrite mais assez universelle stipule qu'une bonne traduction devrait aspirer à la perfection linguistique.

Il est fondamental, d'ailleurs, que les apprenants ne perdent pas de vue les liens entre les cours de langue (très nombreux dans notre département, comme on a vu dans la section 2) et les cours de traduction : il convient d'assurer une intégration des différentes compétences dont un bon traducteur a besoin. 
En résumé, c'est précisément parce que nous ne ressentons pas le besoin de traiter les fautes de langue pendant les cours (dans la mise en commun orale qui suit chaque exercice individuel, nous préférons nous concentrer sur les véritables problèmes de traduction) que nous avons prévu tant de boutons d'ordre linguistique dans notre barre. Les informations fournies de cette façon à l'apprenant sont tellement précises qu'il devient superflu de parler du thème en classe.

\subsection{La cause et l'effet des erreurs et des fautes}

La classification décrite dans la section 3.2 tient compte à la fois des causes et des effets des erreurs et des fautes.

Pendant la correction, nous nous demandons systématiquement si la faille relevée dans le texte de l'étudiant est due à une lacune dans la maîtrise de la langue cible (catégories 1-4) ou s'il s'agit plutôt d'une faute de traduction (catégories 6,8). Cela explique la présence, par exemple, de deux boutons différents relatifs au nombre des substantifs: le code MS signale que l'étudiant a commis une faute morphologique qui n'est pas induite directement par le processus de la traduction (c'est le cas s'il écrit musea's au lieu de museums ou musea, par exemple), pendant que le code EM désigne une interférence entre l'espagnol et le néerlandais (comme pourrait être le calque du singulier gasto corriente).

La présence de la classe de commentaires contrastifs (la catégorie 8) s'explique par notre désir de distinguer entre les « erreurs» attribuables à l'immaturité (naturelle à un certain moment de la formation de l'apprenant) de la compétence traductrice et les «fautes» découlant d'une mise en œuvre insuffisante des compétences acquises. Cette différentiation se reflète dans la pondération des deux catégories de transgressions: la pénalisation associée au bouton STA (problèmes stylistiques de tout genre) est inférieure à celle du bouton NOM (dès le début du cours, les étudiants sont sensibilisés à la nécessité d'effectuer certaines transformations afin d'éviter une reproduction maladroite du caractère nominal de la prose espagnole).

Les boutons LI, HI et ZI correspondent à des erreurs et fautes d'interprétation jugées plus ou moins graves (et qui donnent lieu, en conséquence, à une sanction plus ou moins sévère), compte tenu de l'ampleur des dégâts causés (les méprises locales sont considérées moins catastrophiques que les incorrections dont les effets s'étendent sur plusieurs paragraphes; les contresens et les fautes commises dans la traduction des titres méritent l'amende maximale) et de l'adéquation entre compétence et performance (l'apprenant devrait être capable d'éviter les interférences lexicales répertoriées dans notre environnement électronique; nous sommes plus indulgents quand il se méprend sur des tournures qui n'ont pas fait l'objet d'un enseignement explicite).

D'autre part, c'est seulement dans l'étape de l'évaluation sommative que commence à être vraiment importante la pénalisation attribuée aux différents boutons. Il nous semble important d'éviter une dissociation schizophrénique entre les méthodes et les normes appliquées pendant la phase de l'évaluation formative et celles que l'on utilise dans l'évaluation sommative. N'empêche que nous préférons ne pas exporter les statistiques détaillées dans les premiers stades du processus d'apprentissage. Au fur et à mesure que l'étudiant avance dans ses études, nous lui fournissons un feed-back quantitatif plus exhaustif et précis; un mois avant la fin du cours, nous organisons 
un exercice qui simule les circonstances de l'examen final, et c'est à ce moment que nous donnons une première note.

\subsection{La correction comme tremplin}

Dans le stade de l'évaluation formative, la correction devrait revêtir un caractère dynamique. Il ne suffit pas de formuler un arrêt définitif sur la qualité du produit délivré par l'apprenant, ni de lui proposer une alternative correcte pour chaque inexactitude de son texte. Chacune des annotations longues correspondant aux différents boutons «négatifs » contient un hyperlien qui mène à une page web consacrée au problème soulevé par le passage en question.

L'apprenant y trouve d'abord une description détaillée de la difficulté, avec des exemples concrets qu'il peut collationner avec le fragment qu'il a traduit lui-même. Il est donc obligé de situer chaque cas concret dans un cadre plus ample. Parfois, la page web contiendra une «règle» de traduction très simple (c'est le cas des problèmes contrastifs auxquels correspondent les boutons de la huitième catégorie), parfois il s'agira plutôt d'une invitation à la réflexion (par exemple, dans les pages consacrées au «Skopos»).

Dans tous les cas, nous avons essayé de créer une page «dynamique», en ajoutant des suggestions d'activités de remédiation. Celles-ci peuvent adopter la forme d'une simple référence à un chapitre spécifique des manuels d'orthographe ou de style publiés par nos collègues des cours de néerlandais, ou à la batterie d'exercices de néerlandais qui sont à la disposition de nos étudiants. Pour les problèmes proprement traductologiques, nous invitons les étudiants à consulter des ouvrages spécialisés, à participer à un échange d'opinions sur le forum de l'environnement électronique (où ils peuvent raisonner les stratégies et les techniques qu'ils ont appliquées) ou à faire des recherches personnelles. Ainsi, nous leur demandons d'explorer les archives de quelques journaux espagnols et belges, de créer leur propre corpus d'articles et d'étudier certaines conventions du genre. Cela leur permet de découvrir, par exemple, les différences entre les titres espagnols (lesquels, généralement, adoptent la forme d'une phrase complète) et les titres employés en néerlandais (bien plus brefs, entre autres à cause de l'omission d'articles et de prépositions). En plus, ils disposent d'un logiciel d'analyse de textes (WordSmith Tools) grâce auquel ils peuvent dépister des informations de type phraséologique qui manquent dans les ouvrages de référence classiques.

Les textes traduits par les élèves se renouvellent chaque année, mais nous essayons de rentabiliser au maximum les exercices faits dans le passé, qui constituent pour nous une mine d'or d'où nous prenons les exemples qui illustrent les explications de la page web. D'ailleurs, les textes sur lesquels ont travaillé les promotions précédentes et nos traductions commentées peuvent être consultés intégralement dans notre environnement électronique, de sorte que les étudiants désireux de multiplier les exercices puissent le faire à cœur joie.

Certes, cette approche n'est pas exempte de risques. Il n'est pas impossible que le caractère très détailliste de nos annotations et les ramifications infinies de la toile électronique finissent par décourager les apprenants, submergés par les ondes interminables du flot d'informations. Afin d'aider les étudiants à structurer leurs efforts, nous ajoutons toujours une appréciation globale. Le menu de Markin permet d'insérer un 
feed-back général de deux types (voir la prise d'écran de l'annexe 1). Nous avons déjà signalé que, dans la phase initiale du cours, nous n'exportons pas les statistiques détaillées fournies par le programme, mais nous partons de celles-ci pour signaler à l'étudiant quels sont les aspects qu'il doit travailler en premier lieu. Très souvent, dans le feed-back 1, nous essayons de mettre à l'aise les apprenants, en contrebalançant le nombre souvent très élevé de commentaires spécifiques avec une appréciation générale plus encourageante. (Nous utilisons le feed-back 2 du menu de Markin pour émettre une opinion sur la qualité du cahier des charges et du dossier de documentation qui accompagnent la traduction.)

D'ailleurs, ce n'est pas seulement après coup que nous suggérons aux apprenants d'accorder la primauté à certaines catégories de problèmes. Avant de remettre une tâche, ils doivent parcourir une liste de contrôle et vérifier s'ils ont fait tout le possible pour éviter une série de méprises représentatives (voir l'annexe 4 pour un exemple d'une telle liste).

\section{Projets de recherches}

Il nous semble évident que l'évaluation (formative ou sommative) d'une traduction ne pourra jamais aspirer au statut d'expérience scientifique. La multiplication des catégories de fautes ne garantit pas l'objectivité ou l'intersubjectivité de la correction. Non seulement il s'est avéré difficile d'arriver, au sein de notre département, à un consensus sur la classification et la pondération des différentes erreurs et fautes mais il est aussi vraisemblable que même les professeurs qui se sont mis d'accord sur la méthode à appliquer proposeront parfois des notes très différentes pour la même version.

Néanmoins, la méthodologie appliquée dans la phase formative devrait permettre aux étudiants de faire des progrès mesurables et quantifiables. L'évaluation sommative, d'autre part, devrait être un exercice reproductible au moins dans les circonstances suivantes: si on demande au même professeur de corriger les mêmes traductions plusieurs fois, avec un intervalle de quelques semaines ou quelques mois, il devrait aboutir plus ou moins aux mêmes résultats (en effet, le verdict du professeur ne devrait pas dépendre de son humeur du jour). Selon nos hypothèses, il est plus facile d'atteindre cet objectif si on emploie une grille de correction nuancée et détaillée, au lieu de formuler une appréciation holistique.

Nous avons l'intention d'évaluer, au cours des mois suivants, notre méthode d'évaluation formative et sommative. Au moyen d'une enquête parmi les apprenants et d'un test empirique (dans lequel les performances de nos étudiants seront comparées à plusieurs moments du processus d'apprentissage avec celles d'un groupe de contrôle), nous nous proposons de mesurer le succès subjectif et objectif de notre approche. En plus, en ce qui concerne l'évaluation sommative, nous avons l'intention de refaire, avec les changements qui s'imposent, les expériences décrites par Waddington (1999). Les résultats de ces recherches nous montreront quels sont les aspects de notre méthodologie qu'il convient de peaufiner. 


\section{RÉFÉRENCES}

ConseIL DE L'EuRope (2000): Un cadre européen commun de référence pour les langues: apprendre, enseigner, évaluer, <http://www.culture2.coe.int/portfolio/> (il existe une version imprimée commercialisée par les Éditions Didier).

Delisle, J., Lee-Jahnke, H. et M.C. Cormier (eds.) (1999) : Terminologie de la traduction, Translation Terminology, Terminología de la traducción, Terminologie der Übersetzung, Amsterdam, John Benjamins Publishing Company.

Delisle, J., Lee-Jahnke, H. Cormier, M.C., Bloemen, H. and W. Segers (trad. et adapt.) (2003): Terminologie van de vertaling, Nijmegen, Vantilt.

House, J. (1977): A Model for Translation Quality Assessment, Tübingen, Narr.

House, J. (1997): Translation Quality Assessment: A Model Revisited, Tübingen, Narr.

House, J. (2001) : «Translation Quality Assessment: Linguistic Description versus Social Evaluation», META 46-2, numéro spécial «Évaluation: Paramètres, méthodes, aspects pédagogiques».

Hulst, J. (1998): «Het product centraal: criteria en methoden voor de evaluatie van vertalingen », De kracht van vertaling (H. Bloemen et J. Hulst, dir.), Utrecht, Platform Vertalen en Vertaalwetenschap.

Lee-Jahnke, H. (2001): "Aspects pédagogiques de l'évaluation en traduction», META 46-2, numéro spécial «Évaluation: Paramètres, méthodes, aspects pédagogiques».

Meta (2000) : 46-2, numéro spécial «Évaluation: Paramètres, méthodes, aspects pédagogiques».

Translator, The (2000): 6-2, numéro spécial «Evaluation and Translation» (Carol Maier, guest ed.), Manchester, St. Jerome.

WAdDington, C. (1999): Estudio comparativo de diferentes métodos de evaluación de la traducción general (Inglés-Español), Madrid, Publicaciones de la Universidad Pontificia Comillas.

\section{ANNEXES}

1. Prise d'écran: menu du logiciel Markin.

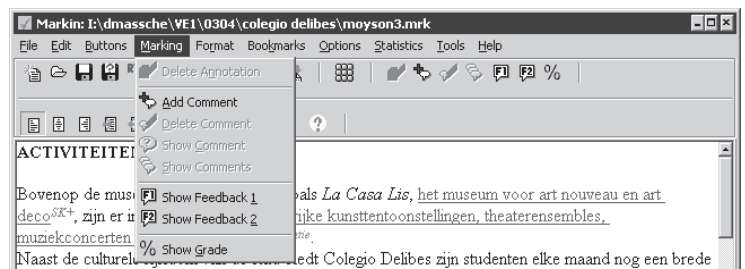

2. Prise d'écran: barre de boutons créée pour la correction des traductions de l'espagnol vers le néerlandais de textes généraux ou plus spécialisés. Cette barre de boutons (avec l'explication des codes, les annotations courtes et longues, la pénalisation, les hyperliens, etc.) peut être téléchargée dans notre environnement électronique (http://blackboard.vlekho.wenk.be), auquel les intéressés pourront avoir accès sur simple demande (dmassche@vlekho.wenk.be).

\begin{tabular}{|c|c|c|c|c|c|c|c|c|c|c|}
\hline \multicolumn{11}{|c|}{ spnl dm } \\
\hline 01 & wW & AE & HL & WT & TL & AS & 02 & LT & 03 & RV \\
\hline LEX & oc & PP & BV & AV & ov & LW & vB & $\mathrm{vz}$ & ww & GE \\
\hline MS & VA & $\mathrm{cV}$ & TE & co & WTI & BB & $m$ & vs & $\mathrm{oz}$ & NO \\
\hline SYA & 04 & TR & $\mathrm{CH}$ & OP & LZ & oo & TP & STA & 05 & TF \\
\hline CIJ & AK & II. & EO & ST & TA & 06 & or & ow & ODV & wK \\
\hline $\mathrm{u}$ & z। & HI & SK1 & SK2 & Sк3 & ICS & If & 07 & $\mathrm{sc}$ & PT \\
\hline DB & 08 & NOM & ss & EM & AG & NS & OA & REL & BIJ & CA \\
\hline SE & SG & BM & $A A$ & PVG & PVP & PV & GER & IND & SES & MP \\
\hline \multicolumn{2}{|c|}{ MSU MAD } & TV & PR & CJ & PPr & HER & & & & \\
\hline $\mathrm{T}+$ & S+ & $1+$ & SK & $\mathrm{CO}+$ & D+ & C. & & & & \\
\hline
\end{tabular}


3. Prise d'écran: définition du bouton SK2, avec le code d'erreur, une annotation courte, une annotation longue, etc.

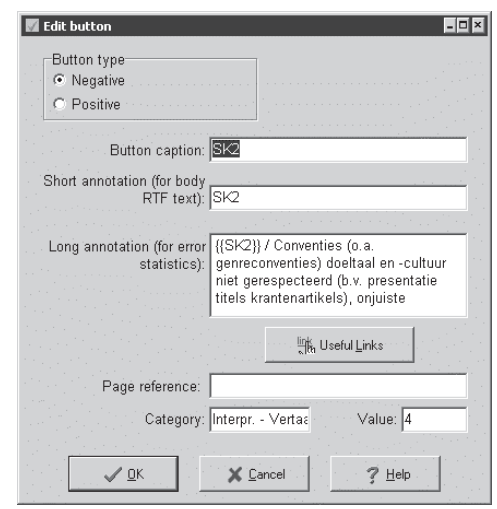

4. Liste de contrôle

a) Je n'ai quand même pas écrit de point après mon titre?

b) La subdivision du texte en alinéas est-elle adéquate?

c) Est-ce que j'ai respecté les conventions en ce qui concerne la présentation des chiffres?

d) Est-ce que je n'ai pas oublié de vérifier mon texte à l'aide des outils du traitement de texte?

e) Est-ce que j'ai exploité correctement la documentation disponible dans l'environnement électronique (par exemple la base de données)?

f) Ne serait-il pas possible d'éviter la construction 'article + infinitif' (trop nominale)?

g) Ne serait-il pas possible d'éviter les formes du participe présent (il y a beaucoup d'autres solutions pour la traduction du gerundio)?

h) Est-ce que je comprends mon propre texte?

i) Ma traduction est-elle bien ajustée au «Skopos»?

j) Je n'ai quand même pas commis mes trois fautes favorites [trois fautes à sélectionner par l'étudiant dans les catégories de la barre de boutons]? 